\title{
Glutathione disulfide induces apoptosis in U937 cells by a redox-mediated p38 MAP kinase pathway ${ }^{1}$
}

\author{
GIUSEPPE FILOMENI, GIUSEPPE ROTILIO, AND MARIA ROSA CIRIOLO*,2 \\ Department of Biology, University of Rome "Tor Vergata," Rome, Italy; and *Department of \\ Biomedical Sciences, University of Chieti "G. D’Annunzio," Chieti, Italy
}

\section{SPECIFIC AIM}

Experimental evidence has documented the involvement of oxidation reduction (redox) reactions in the regulation of key molecular mechanisms linked to important cellular functions. The aim of our research was to identify the molecular mechanism(s) underlying cell signaling leading to cell cycle alteration on an oxidative insult mediated by glutathione disulfide in U937 cells.

\section{PRINCIPAL FINDINGS}

\section{GSSG commits U937 cells to apoptosis}

GSSG treatment leads to a dose-dependent induction of U937 cell death. Microscopic examination of Hoechst 33342 staining revealed that U937 cells treated with GSSG have the characteristic features of apoptotic nuclei. Additional cytofluorometric analyses showed a time-dependent increase in annexin $\mathrm{V}$ and propidium iodide positive cells indicating that apoptotic cells underwent secondary necrosis. Western blot analyses of cytosolic cytochrome $c$ and active caspase-9 showed an increase in the immunoreactive bands at $18 \mathrm{~h}$ on $1 \mathrm{mM}$ GSSG treatment whereas fluorometric analyses of caspase-3 activity was significantly different from untreated cells as early as $24 \mathrm{~h}$ treatment.

\section{GSSG alters cellular redox environment}

GSSG treatment results in a significant dose-dependent decrease of reduced glutathione without a corresponding increase in the disulfide form of the tripeptide. The decrement observed was due to engagement of GSH in the formation of mixed disulfides with intracellular protein thiols. Since GSSG per se is a nonmembrane permeable molecule, it must exert its toxic effects by oxidizing specific cysteine residues on the external side of the plasma membrane. On incubation with Alexa fluor $\mathrm{C}_{5}$ maleimide, a significant decrease in the fluorescence of cells treated with $1 \mathrm{mM} \mathrm{GSSG}$ was detected, indicating that cysteine residues of membrane-associated proteins were not available for reaction with the fluorescent probe. The imbalance in the redox homeostasis results in an increased flux of reactive oxygen species (ROS) detected by DCF-DA fluorophore and assayed by FACS analysis. U937 cells treated with GSSG had markedly high levels of DCF fluorescence; this increase was significantly different from untreated cells at $12 \mathrm{~h}$ of treatment, when GSH starts to decline. $1 \mathrm{mM}$ GSSG treatment also results in a time-dependent Bcl-2 protein down-regulation starting as early as after $3 \mathrm{~h}$ of treatment. This can be a detrimental factor both for cell survival and ROS generation. Thioredoxin, another component of the redox couples involved in intracellular redox homeostasis, was also affected on GSSG treatment. A comparison of thioredoxin immunoreactive levels under nonreducing conditions demonstrated that $1 \mathrm{mM}$ GSSG treatment induced a decrease in the content of its reduced form $(-63 \pm 5 \%)$. Immunoprecipitations performed using a polyclonal antibody against ASK1 showed that thioredoxin content was significantly decreased, suggesting that GSSG-mediated toxicity could be transduced through this upstream activator of mitogen activated protein (MAP) kinases.

\section{Apoptosis was abolished by restoring the intracellular redox buffer}

Treatment with GSHest, a permeable compound able to increase the intracellular content of GSH, prevents GSSG-induced cell death (Fig. $1 A, D)$ by maintaining a significantly high GSH content, with values close to that observed in control cells (Fig. 1B). Hallmarks of apoptotic program such as Bcl-2 down-regulation, cytochrome $c$ release, and caspase-9 proteolytic activation were inhibited (Fig. 1C). Concomitantly, thioredoxin redox state and ASK1/thioredoxin association were partially restored, suggesting that GSHest inhibited the commitment of cell death by blocking the early stages of GSSG-mediated redox-dependent signal transduction.

\footnotetext{
${ }^{1}$ To read the full text of this article, go to http://www. fasebj.org/cgi/doi/10.1096/fj.02-0105fje; to cite this article, use FASEB J. (November 1, 2002) 10.1096/fj.02-0105fje

${ }^{2}$ Correspondence: Department of Biomedical Sciences, University of Chieti “G. D’Annunzio,” Via dei Vestini 66013, Chieti, Italy. E-mail: ciriolo@bio.uniroma2.it
} 

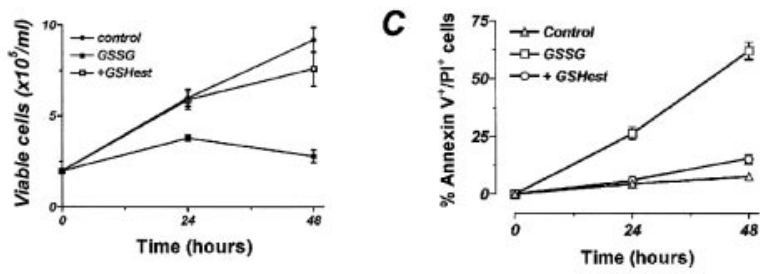

B

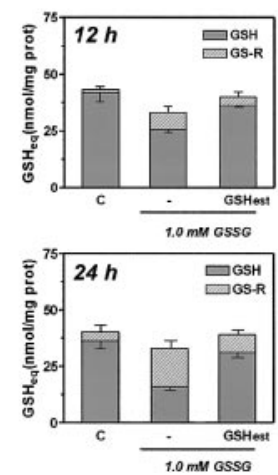

D

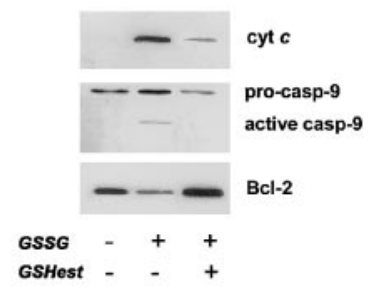

affected by the addition of SB 203580 (Fig. 2C). GSHest pretreatment was also able to inhibit MKK3/6 and p38 MAP kinase phospho-activation, indicating that GSSGinduced redox changes were fundamental to p38 MAP kinase pathway activation.

\section{GSSG do not affect viability and intracellular redox state of primary monocytes}

One approach used to selectively kill tumor cells is represented by alteration of the intracellular redox state; under physiological conditions, differentiated cells possess more efficient antioxidant defenses with respect to tumor cells. GSSG treatment up to $5 \mathrm{mM}$ induced neither loss of viability nor a decrease in the intracellular GSH concentration in primary monocytes or phorbol-12-myristate-13-acetate (PMA) -differenti-

$\boldsymbol{A}$

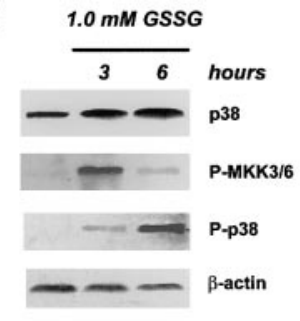

C calculated using WinMDI version 2.8 software. D) U937 cells were treated with $1 \mathrm{mM}$ GSSG for $18 \mathrm{~h}$ and used for Western blot analyses. $20 \mu \mathrm{g}$ of proteins from cytosolic extract was loaded onto each lane for cytosolic cytochrome $c$ and caspase-9, $20 \mu \mathrm{g}$ of proteins from total cell lysates for Bcl-2. Results are from 1 experiment representative of 3 that gave similar results. Data reported in panels $A, C$ are from 3 separate experiments performed in duplicate and expressed as means \pm SD. GSSG: cells treated with $1 \mathrm{mM}$ GSSG; + GSHest: cells incubated with $1 \mathrm{mM}$ GSH ethyl ester $1 \mathrm{~h}$ before and during GSSG treatment.

\section{GSSG induces cell death by the p38 MAP kinase pathway}

p38 MAP kinase pathway has well-established roles in the regulation and function of proinflammatory cytokines; it is activated in cultured cells as ASK1 downstream kinase in events mediated by membrane receptors and stress. Figure $\boldsymbol{2} \boldsymbol{A}$ shows that p38 MAP kinase pathway was activated after treatment with $1 \mathrm{mM} \mathrm{GSSG}$ : upstream MKK3/ 6 were phosphorylated at $3 \mathrm{~h}$ whereas phospho-p38 was activated on $6 \mathrm{~h}$ (Fig. 2A). Treatment of cells with $5 \mu \mathrm{M}$ SB 203580, a pyridyl imidazole compound that specifically inhibits p38 MAP kinase activation, abrogates GSSG-mediated toxicity (Fig. 2B). Cytofluorometric analyses of U937 cells treated with 1 mM GSSG in the presence of SB 203580 show similar profile of untreated cells. Western blot analyses of the molecular factor mediating apoptosis via the mitochondrial pathway showed that Bcl-2 down-regulation, cytochrome $c$ release, and caspase-9 activation were less

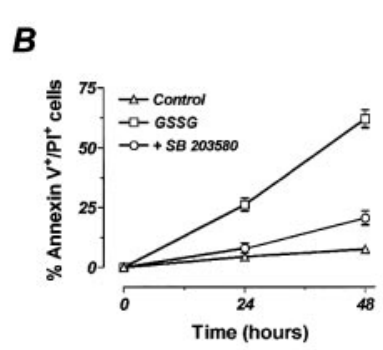

D

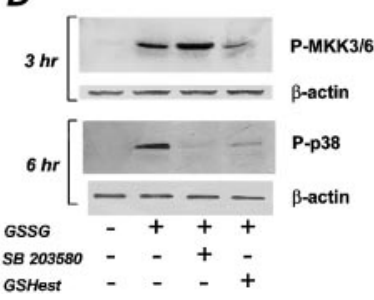

Figure 2. p38 MAP kinase signaling pathway is involved in GSSG-induced apoptosis. A) U937 cells were treated with $1 \mathrm{mM}$ GSSG. Aliquots of cells suspension were withdrawn and treated for Western blot analyses. $50 \mu \mathrm{g}$ of proteins from total cell extract was loaded onto each lane. Results are from 1 experiment representative of 3 with similar results. B) U937 cells were grown in 10\% FBS RPMI; $5 \mu \mathrm{M}$ SB 203580 was added $2 \mathrm{~h}$ before addition of GSSG and maintained throughout. After 24 and $48 \mathrm{~h}$ of treatment with $1 \mathrm{mM}$ GSSG, cells were washed with PBS and stained with annexin V-FITC and propidium iodide for FACScan analyses. GSSG: cells treated with 1 mM GSSG; + SB 203580: cells incubated with $5 \mu \mathrm{M}$ SB $2035802 \mathrm{~h}$ before and during GSSG treatment. Percentages of staining-positive cells were calculated using WinMDI version 2.8 software. Data are from 3 separate experiments carried out in duplicate and expressed as means \pm SD. C) U937 cells were treated with $1 \mathrm{mM}$ GSSG for $18 \mathrm{~h}$. Cell lysates $( \pm$ SB 203580$)$ were analyzed by Western blot analyses. $20 \mu \mathrm{g}$ of cytosolic proteins was loaded onto each lane for cytosolic cytochrome $c$ and caspase-9, $20 \mu \mathrm{g}$ of proteins from total cell lysates for Bcl-2. Results are from 1 experiment representative of 3 that gave similar results. D) $1 \mathrm{mM}$ GSHest or $5 \mu \mathrm{M}$ SB 203580 was added 1 and $2 \mathrm{~h}$ before the addition of GSSG, respectively, and maintained throughout. At 3 and $6 \mathrm{~h}$, aliquots of cells suspension were withdrawn and treated for Western blot analyses. $20 \mu \mathrm{L}$ of sample was loaded onto each lane. Results are from 1 experiment representative of 3 that gave similar results. 


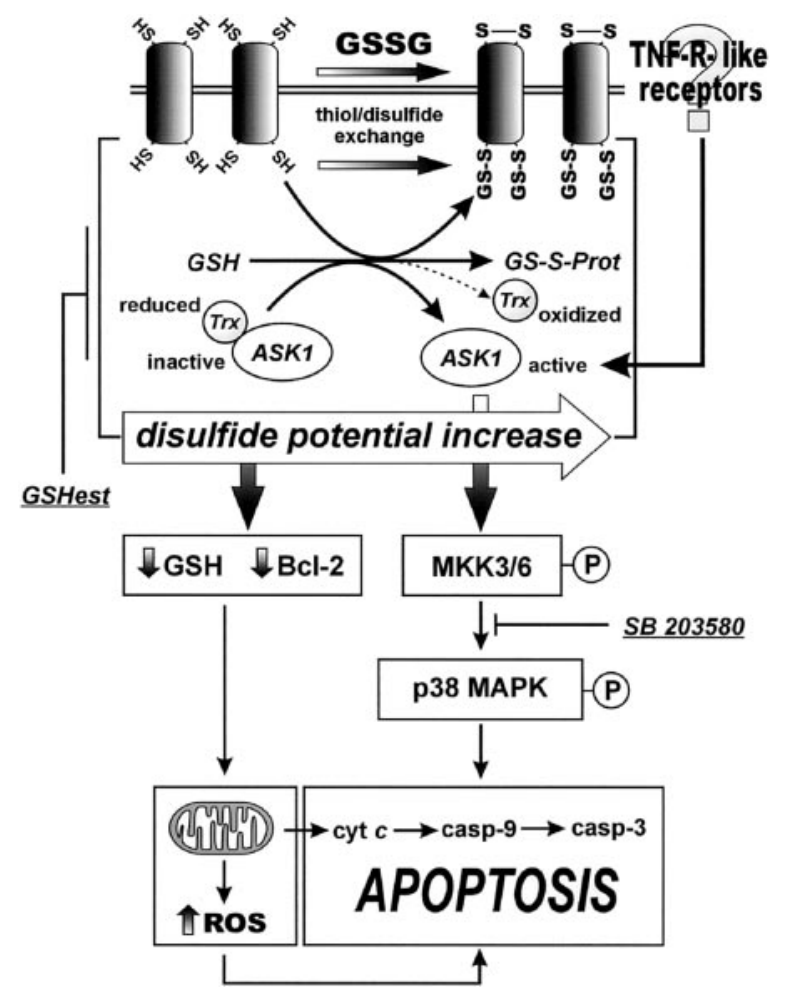

Figure 3. Schematic diagram of the effects of GSSG on U937 cells.

ated U937 cells. The lack of GSSG toxicity in primary or differentiated cells implies the potential clinical efficacy of GSSG in the anti-cancer chemotherapy.

\section{CONCLUSION}

In the current investigation, we have shown that GSSG can induce changes in the intracellular redox state of U937 cells. These GSSG-derived effects participated in cell signaling events that regulated induction of programmed cell death via the mitochondrial pathway. Since GSSG is membrane impermeant, we hypothesized that it can transduce signals via thiol/disulfide exchange with cysteine-rich proteins spanning the cell membrane. This phenomenon was associated with a dramatic alteration in the intracellular redox state of thiols toward oxidizing conditions that were not due to an increase of GSSG but rather to formation of mixed disulfides of GSH with protein thiols. An increase in ROS production was detected after $12 \mathrm{~h}$ of treatment, which corresponds to when the GSH decrease was significant. This points to a sequence in the events downstream of GSSG treatment wherein ROS increase is simultaneous with the GSH decrement and subsequent to the earlier decrease in expression of the antiapoptotic protein Bcl-2. We have shown that the GSSG-induced signal transduction takes place through the p38 MAP kinase cascade activation. Activation of this pathway was observed before the changes in GSH homeostasis, indicating that the p38 MAP kinase pathway sensed very low changes of the intracellular redox state. On the other hand, the decrement in GSH content could be an amplifying cofactor of p38-induced cell death that, by keeping the intracellular environment in an oxidative state, favors the execution step of programmed cell death. Under our experimental conditions, thioredoxin redox state was altered on GSSG treatment. Moreover, the concomitant decrease of its immunoreactive band, on coprecipitation with ASK1, indicated an involvement of thioredoxin/ASK1 complex in the GSSG-mediated oxidative insult. Even if this mechanism remained to be totally elucidated and is currently under investigation, a direct relationship between thioredoxin, ASK1 and GSSG-mediated oxidative stress seems to be reasonable in our experimental model. The involvement of p38 MAP kinase activation pathway in GSSGinduced U937 cell death was confirmed by the protection achieved with GSHest and the p38 inhibitor SB 203580. GSHest treatment also affected the thioredoxin/ASK1 complex, suggesting that its protective effect was upstream respect to that exerted by SB 203580. In fact, p38 MAP kinase pathway was inhibited at the level of MKK $3 / 6$, even though a direct inhibition of p38 MAP kinase by GSHest could not be excluded. However, especially in experiments carried out in the presence of p38 inhibitor, we noticed that a small amount of cells still underwent apoptosis. It is conceivable that the redox alterations induced by GSSG, which became severe after $24 \mathrm{~h}$, were able to induce cell death by mechanisms not related to activation of p38 MAP kinase pathway.

Therefore, without crossing the cell membrane, GSSG externally added was able to trigger cell death with the mode of apoptosis by activation of a canonical oxidative stress response, via alteration of the intracellular GSH/ GSSG redox buffer system, and increased ROS production and, at the same time, it can be envisaged as ligand-like factor. This role is supported by the fact that, during the cellular response to GSSG, several molecular markers of the receptor-mediated pathway, such as those that belong to p38 MAP kinase-mediated signaling are activated. Although the characterization of the membrane proteins, which are potential target of GSSG, is still under investigation in our laboratory, we can suggest that ASK1 upstream cysteine rich receptors, such as those belonging to the TNF-R superfamily, could be affected. This hypothesis is strengthened by the lack of effects in intracellular redox state or in cell viability on primary monocytes or PMA-differentiated U937 cells treated with GSSG, and is in line with the finding that, tumor cells are generally more sensitive to the detrimental effects mediated by specific TNF receptors such as by TNF-related apoptosis inducing ligand (TRAIL) receptor. By specifically altering the intracellular redox state of tumor cells without effects on differentiated cells, GSSG may be regarded as a potential tool for enhancing cell sensitivity to chemotherapeutic agents or radiation. 